\title{
JOINT POWER, RATE, AND CHANNEL ALLOCATION IN MULTILINK (COGNITIVE) RADIO SYSTEM
}

\author{
Peter Larsson, Jonas Kronander \\ Ericsson Research, Ericsson AB, 16480 Stockholm, Sweden. \\ peter.j.larsson I jonas.kronander@ericsson.com
}

\begin{abstract}
We consider multi-constrained power, rate and channel allocation crafted for low power consumption, delay tolerant traffic, and under interfering link conditions that may be used in a cognitive radio system. Specifically, an iterative distributed algorithm, based on a sum-power constrained sum-rate maximization with upper (and lower) per user and channel power and rate constraints, as well as upper per user sum-power and sum-rate constraints is developed. The feasibility and performance of the algorithm is demonstrated by simulation in a cellular system. Simulations show that the multiple constraints are handled while improving the sum-rate vs. sum-power relative an "equal power adaptive rate" RRM approach.
\end{abstract}

Index Terms - Power control, rate control, channel allocation, RRM, cognitive radio.

\section{INTRODUCTION}

Nowadays, cellular systems do not just carry voice traffic, but rather a range of traffic classes with different requirements. Delay tolerant (DT) (or best effort) traffic is one central component due to the increased use in data traffic. Classical power control (PC) schemes have primarily aimed for fixed rates, suitable for voice, but that is not needed here. Thus, it is of interest to look into radio resource management $(R R M)$ for DT traffic, [1][2][3].

Another factor is that efficient power (and energy) use is of interest for cellular system operators, vendors and society. This has to do with the desire to offer a green and energy efficient communication, reduced electricity and hardware costs, get optimal performance for the invested power, and extended mobile phone battery time.

Yet another RRM based motivation relates to cognitive radio $(C R)$ systems which can access primary user spectrum as long as they do not too severely harm the communication of primary users, see e.g., [4].

In previous papers related joint power and rate control schemes have been developed and analyzed; in [5] an RRM scheme for DT traffic situations was developed,

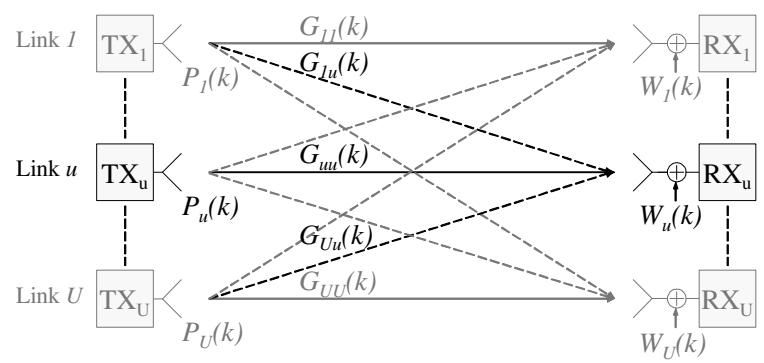

Figure 1. System model with $U$ links for a channel $k$ assuming a single channel where the system sum-rate is maximized under a sum-power constraint, while including a max rate and max power for the channel. In [6], this work was extended to include scheduling and fairness. In contrast to this, CR generally assumes multiple channels.

This paper extends the work and the iterative distributed algorithm in [5] to multiple channels, includes additional per user rate and per user power constraints over the multiple channels, and motivates the approximate optimization criteria used in [5] for cellular systems. We evaluate the viability and performance of our algorithm by simulation in a cellular setting where the CR aspect results in temporarily borrowing primary user spectrum. It is found that all algorithm extensions works as desired and that the sum-rate vs. sum-power performance exceeds a "fixed (equal) power adaptive rate" (FPAR) method.

\section{SYSTEM MODEL}

We consider a wireless system with $U$ interfering links with predefined TX-RX pairs, each link having access to the same $K$ orthogonal channels. Assuming circular symmetric complex Gaussian signals and noise, with variance $P_{u}(k)$ and $W_{u}(k)$ respectively, the per user $u$ per channel $k$ link rate is modeled by the bandwidth normalized Shannon capacity

$$
R_{u}(k)=\ln \left(1+\Gamma_{u}(k)\right),
$$

in nats, where

$$
\Gamma_{u}(k)=\frac{P_{u}(k) G_{u u}(k)}{W_{u}(k)+\sum_{\forall j \neq u} P_{j}(k) G_{j u}(k)}
$$


is the signal to interference and noise ratio (SINR) for link $u$, and $G$ denote path gains. The indices $u$ and $j$ denote the studied and all other links, respectively.

\section{POWER, RATE, AND CHANNEL ALLOCATION}

\section{A. Optimization objectives}

We wish to formulate a very general power, rate and channel allocation framework that can account for multiple power and rate constraints while exploiting the invested total system power in an efficient manner. This is desired as different senders have different needs, capabilities, regulatory constraints and we want to design a power efficient radio system. To avoid the NPcompleteness commonly seen in (per user) channel allocation problems, we integrate the channel allocation in the power and rate control, such that channels are seen as allocated for positive powers. The power efficiency, and the integration of channel allocation, is catered for by an overall optimization objective that strive to maximize system sum-rate over all users and all resources for any invested sum-power over all users and all resources, $P_{t o t}$. We also impose a HW and battery related limit for each user, which translate to a sum-power limit over all resources, $P_{u}^{\max }$. As the data rate for real services are generally upper limited in practice, the sum-rate over all resources for a user should not exceed some sum-rate limit, $R_{u}^{\max }$. In order to limit interference to active links, say primary users in a cognitive radio $(C R)$ radio system, the per channel power must also be limited by $P_{u}^{\max }(k)$. Lastly, practical modulation put an upper bound, $R_{u}^{\max }(k)$, on the used per channel rate. We assume that all lower limits are set to zero. To expand on the CR part, the power mask as proposed in [7] may be based on restrictions imposed by primary users, obtained e.g. via spectrum sensing, e.g., [8], and possibly regulatory limits, see Figure 2. It specifies the maximal permitted transmission power $P_{u}^{\max }(k)$ for the CR node $u$ in the frequency band or channel $k$. We now write our optimization problem as

$$
\begin{array}{ll}
\max & R \equiv \sum_{u=1}^{U} \sum_{k=1}^{K} R_{u}(k) \\
\text { s.t. } & P \equiv \sum_{u=1}^{U} \sum_{k=1}^{K} P_{u}(k) \leq P_{\text {tot }}, \\
& 0 \leq \sum_{k=1}^{K} P_{u}(k) \leq P_{u}^{\max }, 0 \leq \sum_{k=1}^{K} R_{u}(k) \leq R_{u}^{\max }, \\
& 0 \leq P_{u}(k) \leq P_{u}^{\max }(k), 0 \leq R_{u}(k) \leq R_{u}^{\max }(k) .
\end{array}
$$


$G_{u u}(k)>G_{u u^{\prime}}(k)$. Thus, the terms in the sum on the right hand side of (6) may be neglected that results in

$$
\frac{W_{u}(k)+\sum_{j=1}^{U} G_{j u}(k) P_{j}(k)}{G_{u u}(k)}=\frac{1}{\lambda} .
$$

Equation (7) will be referred to as the approximate optimisation criteria and is interpretated as a per user and per channel water-filling, connected by interference and $\lambda$. In section IV.A, its validity will be investigated.

\section{An iterative solution to the approximate opt. criteria}

An iterative algorithm solving (7) for a single channel was suggested in [5], which may directly be applied to multiple channels. The total antenna port received power, i.e. the desired, interfering and noise power, is measured and denoted $\hat{P}_{u}^{(m)}(k)$. A parameter $\beta$ is introduced as a convergence control parameter. The $(m+1)^{\text {th }}$ iteration transmit power level is calculated as

$$
P_{u}^{\prime(m+1)}(k)=P_{u}^{(m)}(k)-\beta \Delta \hat{\Pi}_{u}^{(m)}(k),
$$

where

$$
\Delta \hat{\Pi}_{u}^{(m)}(k)=\hat{\Pi}_{u}^{(m)}(k)-\lambda^{-1},
$$

and

$$
\hat{\Pi}_{u}^{(m)}(k)=\frac{\hat{P}_{u}^{(m)}(k)}{G_{u u}^{(m)}(k)} .
$$

Note that the algorithm also requires a lower nonnegative power bound, which is addressed below.

\section{The per user per channel power constraints}

By employing the iterative algorithm (8), negative powers and powers greater than the upper limit, may result. This is resolved by amending power limits to (8) as

$$
P_{u}^{\prime(m+1)}(k)=\min \left(\max \left(P_{u}^{\prime(m+1)}(k), P_{u}^{\min }(k)\right), P_{u}^{\max }(k)\right),
$$

where $P_{u}^{\min }(k)=0$ is used. The operation in (11) can be motivated in the framework of the Lagrange based optimization. Any user that assumes an upper power limit will act as a fixed noise term to other users. This is already handled by (7). The effect of the lower power limit in (11) is that a link that that doesn't meet (7) and need to reduce its transmit power level, will ultimately assume a zero power level. This silencing operation ensures an automatic reuse control method that avoids the need of any preplanned frequency or timeslot re-use. More importantly, this is how a user selects channels. Also, [9] use the approach in (11), but for a slightly different PC goal. Note that per channel convergence of (8) is studied in [5].

An important aspect is that the iterative solution to (4), i.e., (8) with a positive power constraint, controls both the up- and down-ramping of the power based on the selected $\lambda$, unless a lower power value must be adopted to meet the max power limit. The same idea of power down control for exceeded constraints is used for the other constraints.

\section{E. The per user per channel rate constraints}

At closer study, it is revealed that some links get excessive SINR that can not be fully exploited by practical modulation and coding schemes (MCSs). Thus, to ensure real rate limits, the algorithm must be extended with a per user per channel upper rate constraint. We extend the approach developed in [5] to multiple, channels. The basic idea suggested here is to activate a SINR balancing scheme, reducing the per user and per channel power, whenever (8) would provide excessive link rates to not exceeded the maximum rate. Based on (1), the maximum rate condition translates into a maximum SINR condition via $\Gamma_{u}^{\max }(k)=e^{R_{u}^{\max }(k)}-1 . \quad$ Further, assume that $\hat{\Gamma}_{u}^{(m)}(k)=C^{(m)} P_{k}^{(m)}$ is the measured SINR at the receiver, where $C^{(m)}$ is a proportionality factor, stemming from (2). The $(\mathrm{m}+1)^{\text {th }}$ power needed to obtain $\Gamma_{u}^{\max }(k)$ is $\Gamma_{u}^{\max }(k)=C^{(m+1)} P_{k}^{(m+1)}$. Now, assume that the proportionality factor is constant during the next iteration, i.e., $C^{(m)}=C^{(m+1)}$. The ratio of these two relations result in a well known iterative CIR balancing algorithm [9]

$$
P_{u}^{\prime \prime(m+1)}=P_{u}^{(m)}(k) \frac{\Gamma_{u}^{\max }(k)}{\hat{\Gamma}_{u}^{(m)}(k)},
$$

where we have introduced a double prime on the updated power to distinguish it from the previous versions of the updates in the algorithm. We then introduce the step

$$
P_{u}^{\prime(m+1)}(k)=\min \left(P_{u}^{\prime(m+1)}(k), P_{u}^{\prime \prime(m+1)}(k)\right),
$$

ensuring that the max permissible rate is not exceeded.

\section{$F$. The per user sum-power constraints}

Since the core power allocation follows a water filling approach over the channels, a sum-power exceeding the permissible one would require reducing each channels power with the same amount, since they are controlled by the same Lagrange parameter. This may be written as follows. The total power at the $\mathrm{m}^{\text {th }}$ iteration is

$$
P_{u}^{\prime \prime \prime(m)}=\sum_{k=1}^{K} P_{u}^{(m)}(k) .
$$

The number of active channels with non-zero power is

$$
N_{u}^{(m)}=\max \left(1, \sum_{\forall k}\left(P_{u}^{(m)}(k)>0\right)\right) .
$$

The required power levels that will meet the sum power 
constraint, for fixed interference and noise, are

$$
P_{u}^{\prime \prime \prime(m+1)}(k)=P_{u}^{(m)}(k)+\frac{P_{u}^{\max }-P_{u}^{\prime \prime \prime(m)}}{N_{u}^{(m)}},
$$

then the modified per user per channel power becomes

$$
P_{u}^{\prime^{(m+1)}}(k)=\min \left(P_{u}^{\prime^{(m+1)}}(k), P_{u}^{\prime \prime(m+1)}\right) .
$$

\section{G. The per user sum-rate constraints}

In order not to exceed the per user sum-rate constraint, we reduce the power on all channels for a user based on the water-filling idea since this is the most sum-power efficient approach. For water-filling, we are generally interested to find a local Lagrange parameter $\lambda_{u}$ that adapts the powers such that the rate constraint is not exceeded. One transmitter internal iterative approach which has the same form as (12) is derived here.

First, the per user sum-rate for all active channels $k^{\prime}$ is

$$
R_{u}=\sum_{\forall k^{\prime}} \ln \left(1+\frac{P_{u}(k) G_{u u}(k)}{W_{u}(k)+\sum_{\forall j \neq u} G_{j u}(k) P_{j}(k)}\right),
$$

Using the well-known water-filling power allocation

$$
P_{u}(k)= \begin{cases}\lambda_{u}-\frac{W_{u}(k)+\sum_{\forall j \neq u} G_{j u}(k) P_{j}(k)}{G_{u u}(k)} & \text {, if }>0 \\ 0 & \text {,if }<0\end{cases}
$$

in (18), and with some rewriting, we get

$$
e^{R_{u}}=\lambda_{u}^{N_{u}} \prod_{k^{\prime}}\left(\frac{G_{u u}(k)}{W_{u}(k)+\sum_{\forall j \neq u} G_{j u}(k) P_{j}(k)}\right) .
$$

Roughly following the approach for the derivation in (12), assume that the product term is constant, i.e. $e^{R_{u}}=\lambda_{u}^{N_{u}} \cdot c_{u}$. We may then consider the ratio of (20) at two consecutive internal iteration instances $\mu$, and form the ratio between the two to cancel $c_{u}$ and hence get

$$
\lambda_{u}^{(\mu+1)}=\lambda_{u}^{(\mu)} \cdot e^{\frac{R_{u}^{\max }-R_{u}^{(\mu)}}{N_{u}^{(\mu)}}} .
$$

Equation (21), with some initial value for $\lambda_{u}^{(0)}$, is then used together with (19), for each instance of a measured total antenna port power divided by the own link gain, to generate an updated power $P_{u}^{\prime \prime \prime}(\mu+1)(k)$. The updated power is used in (18) to determine an updated estimated sum-rate $R_{u}^{(\mu)}$, and from this (21) is updated until the relative sumrate error is sufficiently small, i.e. $\left|1-R_{u}^{\max } / R_{u}^{(\mu+1)}\right|<\varepsilon$. Once the internal iteration loop has terminated, the powers $P_{u}^{\prime \prime \prime \prime}(\mu+1)(k)$ are used as upper limits in the algorithm to address the sum-rate constraint via

$$
P_{u}^{\prime(m+1)}(k)=\min \left(P_{u}^{\prime(m+1)}(k), P_{u}^{\prime \prime \prime(m+1)}(k)\right) .
$$

\section{NUMERICAL RESULTS}

We will check the validity of the approximate optimization criteria from III.B, that the various constraints are met on their own and together, and study the system performance. We consider a large system with 100 cells (to provide good statistics), assume one-reuse, wrap around, a uniform user distribution (1 user per cell), four channels (in order to enable presenting channel related results), a pathloss exponent of 3.6 (and 2.6), and Rayleigh fading. The $\mathrm{CR}$ power mask assumes for simplicity the same values for all users for each channel.

\section{A. The accuracy of the approximation}

We first turn to the evaluation of how good the approximative optimisation criteria is. We can rewrite the exact optimisation criteria in (6) to

$$
\begin{aligned}
& \frac{W_{u}(k)+\sum_{j=1}^{U} G_{j u}(k) P_{j}(k)}{G_{u u}(k)}= \\
& \left(\lambda-\sum_{\forall u^{\prime} \neq u}\left(\frac{G_{u u^{\prime}}(k)}{W_{u^{\prime}}(k)+\sum_{j=1}^{U} G_{j u^{\prime}}(k) P_{j}(k)}-\frac{G_{u u^{\prime}}(k)}{W_{u^{\prime}}(k)+\sum_{\forall j \neq u^{\prime}} G_{j u^{\prime}}(k) P_{j}(k)}\right)\right)^{-1}
\end{aligned}
$$

We find that the iterative algorithm for the approximate optimisation criteria (7) can be applied to solve the exact optimisation problem if the Lagrange multiplier is replaced with a parameter that is updated in each iteration

$$
\lambda \rightarrow \lambda-\sum_{\forall u^{\prime} \neq u}\left(\frac{G_{u u^{\prime}}(k)}{W_{u^{\prime}}(k)+\sum_{j=1}^{v} G_{j u^{\prime}}(k) P_{j}(k)}-\frac{G_{u u^{\prime}}(k)}{W_{u^{\prime}}(k)+\sum_{j \neq * u^{\prime}} G_{j u^{\prime}}(k) P_{j}(k)}\right) .
$$

We rewrite (23) in a set of system of equations

$$
\begin{aligned}
& \mathbf{x}_{\mathbf{1}}=\mathbf{w}+\mathbf{G p}, \quad \mathbf{x}_{\mathbf{2}}=\mathbf{w}+(\mathbf{G}-\operatorname{diag}(\mathbf{G})) \mathbf{p} \\
& z_{1}(k)=\frac{1}{x_{1}(k)}, \quad z_{2}(k)=\frac{1}{x_{2}(k)} \\
& \mathbf{G}^{T} \mathbf{z}_{1}=\lambda+\left(\mathbf{G}^{T}-\operatorname{diag}(\mathbf{G})\right) \mathbf{z}_{\mathbf{2}}
\end{aligned}
$$

from which we see, due to the uniqueness of each step if $\mathbf{G}$ is full rank, that if a solution for the power vector exists it has to be unique. Hence, any iteratively converging solution is the correct and unique solution. Figure 3 shows that the approximate optimisation criteria for the sum-rate vs sum-power system performance measure is an excellent approximation in practice.

B. Power vs rate allocation for the approx. opt. criteria and per user and per channel power and rate constraints

In [5], it was shown that for the single channel case, the power and rate characteristics for the approximate solution to ( 4 ), i.e., (7) follows a special relation that straightforwardly extend to multiple channels, i.e.

$$
R_{u}(k)=-\lg _{2}\left(1-\lambda P_{u}(k)\right) \text {. }
$$


Running (8) for positive powers and a fixed $\lambda$ gives the scatter plot points in Figure 4 that perfectly follows the theoretical expected result from (26).

Further, complementing (8) for positive powers with the upper power and rate constraint based algorithms in (11) and (12)-(13) respectively with the constraints $R_{u}^{\max }(k)=3 \mathrm{~b} / \mathrm{Hz} / \mathrm{s}$ and some arbitrarily selected per user indentical max power levels for different channels gives the other scatter plot points shown in Figure 4. This illustrates that the per channel rate and power constraints are not exceeded while adhering to the rate vs. power curve based on the approximate optimization objective (7).

Going back to Figure 3, we also include the sum-rate vs sum-power plots for the case where we have an upper per user and per channel rate limit of $R_{u}^{\max }(k)=3 \mathrm{~b} / \mathrm{Hz} / \mathrm{s}$. We note that the over all sum-rate is reduced with the limit.

In Figure 5, we plot the CDF of all allocated rates, per user and per channel, for about $8 \mathrm{~dB} \mathrm{SNR}$ at the cell border, noting that $10 \%$ of the channels are unassigned any power. As each link has 4 channels here, and each fades independently, there is a high probability that not all channels for a link are assigned zero rate. This is evident from the mean channel rate per link CDF, also shown in Figure 5, where $100 \%$ of the links have a non-zero rate.

An interesting aspect here is that if we let the sumpower constraint $P_{t o t}$ be very large, the per user and per channel power and rate limit will dominate and in the end be the only limiting factors. It turns out that the algorithm converge in few iterations and each channel is assigned either max power, max rate or zero rate and power.

\section{Per user upper sum-power and sum-rate constraints}

Considering (8) for positive powers together with the upper sum-power and sum-rate constraint based algorithms in (14)-(17) and (18)-(22) respectively allow us to examine the upper sum-power and sum-rate constraint. In Figure 7 a per channel and a per link rate CDF is shown when $R_{u}^{\max }=8 \mathrm{~b} / \mathrm{Hz} / \mathrm{s}$. It is noted that the mean link rates does not exceed 8 divided by the 4 channels, i.e. 2 b/Hz/s. In Figure 6, a scatter plot over normalized sum-rate vs. the normalized sum-power also shows that no user exceeds its maximum sum-rate or sum-power constraint.

\section{Simulation results of the full optimization problem}

Figure 8 and 9 consider and shows conformance to all constraints. Figure 10 shows that the proposed algorithm exceed the sum-rate vs. sum-power performance relative to a "fixed (equal) power and adaptive rate" (FPAR) [5] scheme under a wide range of conditions, including two

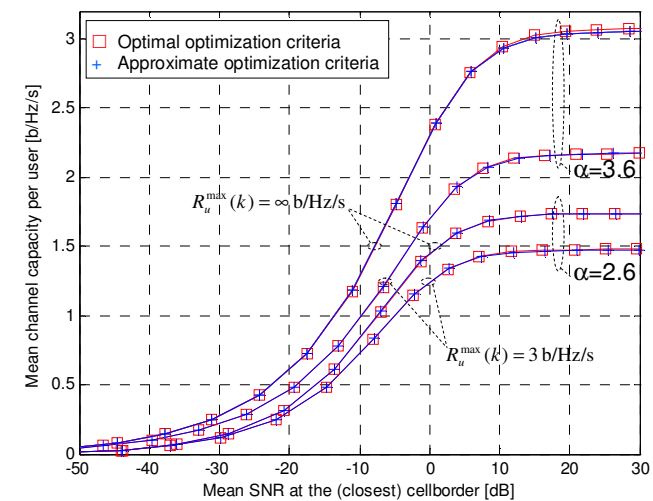

Figure 3. Sum-rate vs. sum-power per channel

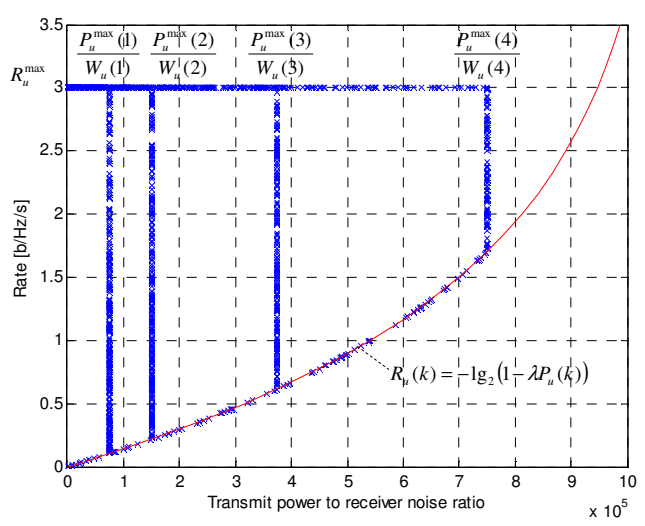

Figure 4. Per channel rate vs. power to noise ratio

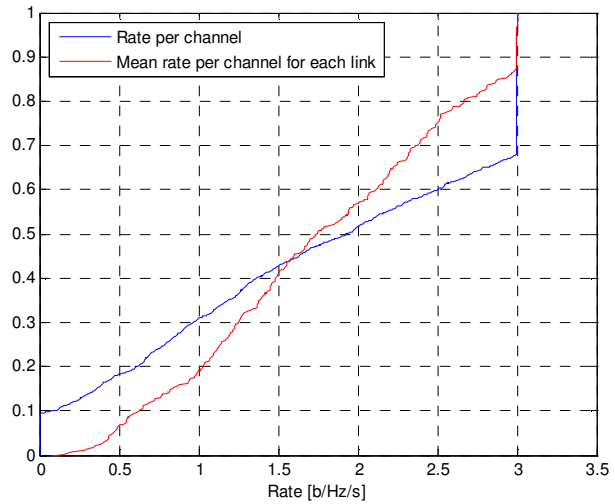

Figure 5. Rate CDFs

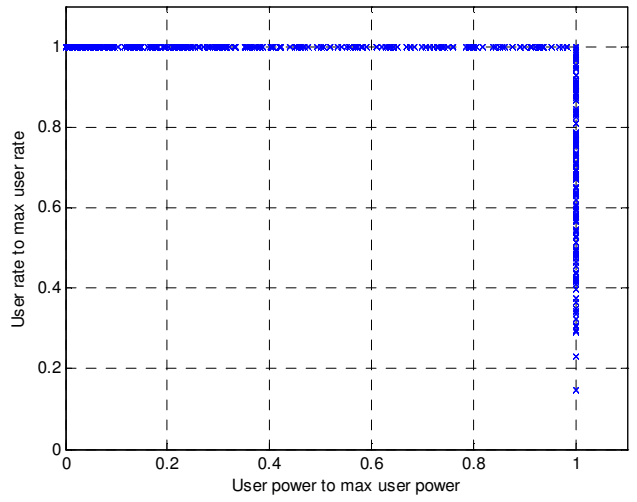

Figure 6 Normalized per user rate vs. per user power 
path loss exponents of 3.6 and 2.6, and a per user and per channel rate and power constraint.

\section{CONCLUSIONS}

In this paper, we introduced a multi-constrained joint power, rate and channel allocation scheme, devised an iterative and distributed algorithm for a cellular or cognitive radio system. We verified that the multiple constraints can be met alone and in combination. We noted that the sum-rate vs. sum-power performance for the studied multi-channel scheme exceeded the performance of a commonly implemented power and rate allocation method for downlink in newer cellular systems of today.

\section{ACKNOWLEDGEMENTS}

This work was carried out within the $7^{\text {th }}$ framework EU program E-Cube. Peter Larsson would also like to thank The Swedish Research Foundation and The Marcus Wallenberg Foundation.

\section{REFERENCES}

[1] F. Berggren, "Power control and adaptive resource allocation in DS-CDMA systems", PhD thesis 2003.

[2] K. Chawla, X. Qiu, "Throughput performance of adaptive modulation in cellular systems", in Proc. IEEE ICUPC, pp. 945-950, 1998.

[3] S.L. Kim, et al., "Combined power control and transmission rate selection in cellular networks", in Proc. IEEE VTC, pp. 1653-1657, Sept. 1999.

[4] S. Haykin, "Cognitive radio: brain-empowered wireless communications," IEEE J-SAC, vol.23, no.2, pp. 201-220, Feb. 2005.

[5] P. Larsson, "Joint power and rate control for delay tolerant traffic in a wireless system," in Proc. IEEE VTC-Spring, pp. 2822-2826, Apr. 2007.

[6] W. Xiaoyu, P. Larsson, "A Joint Power and Rate Control Algorithm and Fairness Enhancement for Multiuser OFDM System," VTC 2008-Fall. IEEE 68th, pp.1-5, 21-24 Sept. 2008.

[7] F. Wang, M. Krunz, S. Cui, "Spectrum Sharing in Cognitive Radio Networks", TR-UA-ECE-2007-1.

[8] S. M. Mishra, A. Sahai, R. W. Brodersen, "Cooperative Sensing among Cognitive Radios", in IEEE ICC Vol. 4, pp. 1658 - 1663, June 2006.

[9] R. Yates, "A framework for uplink power control in cellular radio systems," IEEE J-SAC, vol. 13, no. 7, 1995.

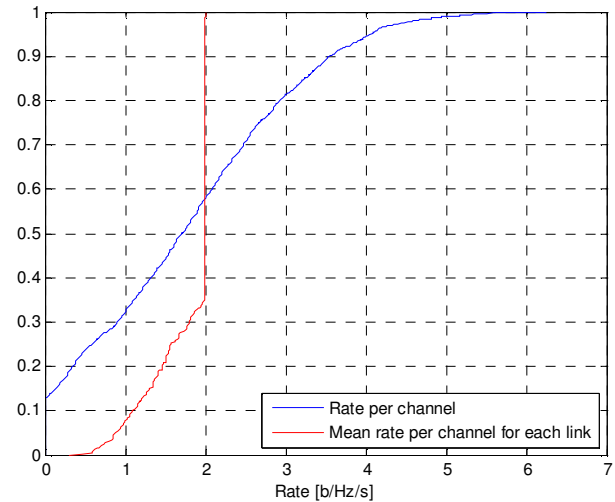

Figure 7. Rate CDFs

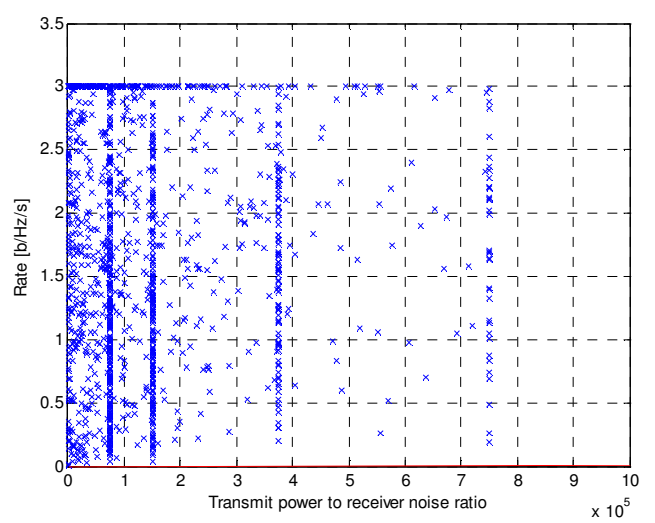

Figure 8. Per channel rate vs. power to noise ratio

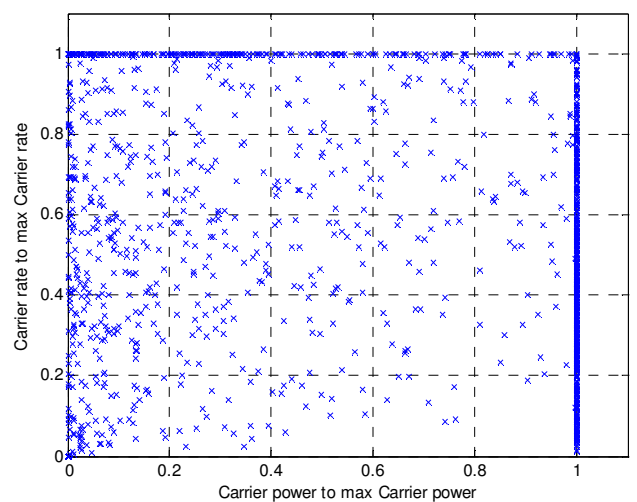

Figure 9. Normalized per user rate vs per user power

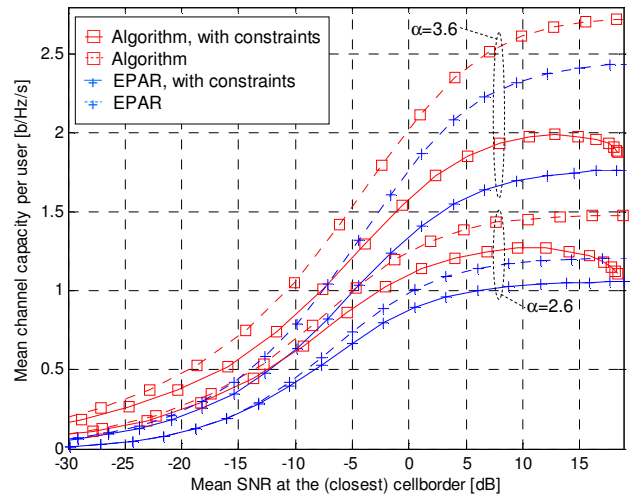

Figure 10. Sum-rate vs. sum-power per channel 